\title{
HIGH SERUM LEVELS OF YKL-40 AND ITS EXPRESSION IN THE MUSCLE TISSUES OF PATIENTS WITH ANTISYNTHETASE SYNDROME
}

\author{
Renata Casseb de Souza Carboni ${ }^{1, *}$, Gustavo Luiz Behrens Pinto ${ }^{1,2}$, Samuel Katsuyuki Shinjo ${ }^{1}$ \\ 1. Universidade de São Paulo, São Paulo (SP), Brazil. 2. Universidade Federal da Bahia, Salvador (BA), Brazil. \\ *Corresponding author: re_casseb@hotmail.com
}

\section{BACKGROUND}

Chitinase-3-like-1 protein (YKL-40) has been associated with many physiological processes, such as inflammation, angiogenesis, cell proliferation, and tissue fibrosis and remodeling. High serum levels of YKL-40 have been described in several systemic autoimmune diseases, but there is still no description of this glycoprotein in antisynthetase syndrome (ASS). Therefore, the aims of the present study were to assess the serum levels of YKL-40 to correlate them with clinical, laboratory, disease status, and therapeutic parameters; and to analyze $\mathrm{YKL}-40$ expression in the muscle tissues of patients with ASS.

\section{MATERIAL AND METHODS}

It is a cross-sectional single-center study that took place from 2017 to 2019, and included 64 adult patients with ASS, who were age-, gender- and ethnicity-matched to 64 healthy control individuals. All patients had presence of the serum autoantibody against aminoacyl-transfer ribonucleic acid synthetase and at least two out of three aspects: joint involvement, muscle and/or lung involvements, in addition to persistent fever at the onset of disease symptoms, Raynaud's phenomenon and/or “mechanic's" hands. The serum levels of the YKL-40 analysis were performed via an ELISA kit method, whereas YKL-40 expression in muscle tissues was analyzed via immunohistochemistry technique. The disease status was assessed using the International Myositis Assessment and Clinical Studies Group (IMACS) set scores.

\section{RESULTS}

The mean age of the patients was $41.5 \pm 12.8$ years, and the median disease duration was $1.5(0.0-4.0)$ year. The study featured predominantly female gender (82.8\%) and Caucasian ethnicity (73.4\%) - data comparable to control group. Most ASS patients had stable disease status according to the IMACS set scores. The median serum levels of YKL-40 were significantly higher in the patients with ASS compared with the healthy individuals: 538.4 (363.4-853.1) pg/mL vs. 270.0 (201.8-451.9) pg/mL, respectively; $p<0.001$. However, the YKL-40 did not correlate to any clinical, laboratory, disease-status, and therapeutic parameters ( $p>0.050)$, except for the serum levels of tumor necrosis factor (TNF)- $a$ (Spearman correlation, rho $=0.382 ; p=0.007$ ). YKL-40 was highly expressed via inflammatory cells (specifically macrophages) found in muscle biopsy samples.

\section{CONCLUSION}

High serum levels of YKL-40 were observed in patients with ASS and correlated positively with serum levels of TNF-a. Moreover, $\mathrm{YKL}-40$ was expressed in muscle tissues mainly through macrophage cells. These data suggest possible $\mathrm{YKL}-40$ involvement in the pathogenesis of ASS.

\section{FUNDING}

This study was funded by Fundação de Amparo à Pesquisa do Estado de São Paulo (FAPESP Grant No. 2014/09079-1 to SKS). 